\title{
Editorials
}

\section{Learning from others}

Jan M. Davies MD FRCPC

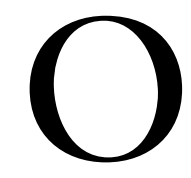

VER the past 157 years, the specialty of Anesthesia has made impressive improvements in safety. These improvements have resulted from a number of different factors, which include:

- invention of the hypodermic syringe and needle (in 1853);

- development of tracheal intubation (in 1880);

- measurement and recording of intraoperative blood pressure (1901); and

- design of the first electronic computer (1943). ${ }^{1}$

Each of these factors is important and has contributed to better care and better outcomes for patients. Each listed above is also important in that the improvement came from outside the specialty of anesthesia. For example, (blind oral) tracheal intubation was developed in 1880 by a Glasgow surgeon, William Macewen, a former student of Lister and pioneer neurosurgeon. ${ }^{1-3}$ The first electronic computer, ENIAC, was developed to calculate artillery firing tables. ${ }^{1}$ But despite their inventors and origins, these improvements have been adopted by anesthesiologists, in some instances almost as their own.

Another example of the adoption of a technique from a different industry is that described by Fasting and Gisvold in this issue of the Journal. ${ }^{4}$ They describe their use of the technique of statistical process control (SPC) for four adverse events occurring during anesthesia: difficulties with intubation, inadequate regional (brachial plexus) anesthesia, drug errors, and difficulties with emergence from general anesthesia. Although the authors have routinely collected data on all anesthetics in their department in Trondheim, Norway for 18 years, ${ }^{5}$ they applied SPC only to the past five years of prospectively recorded data.

\section{What is SPC?}

SPC is a term made up of three quite precisely used words. 'Statistical' refers to having to do with numbers and drawing conclusions from numbers. 'Process' refers to an activity (in contrast to statistical quality control or SQC which is concerned with the characteristics of a product). 'Control' refers to keeping something within boundaries or regulating the activity so that its outcome can be predicted with some degree of accuracy. ${ }^{6}$

Thus, SPC is the use of specific statistical methods to analyze a process and its outcome. SPC allows workers to take appropriate actions to achieve and maintain the process within certain limits, that is, in a state of statistical control. As a result, workers are then able to continuously improve that process. ${ }^{7}$

SPC was first described by Walter A. Shewhart, an American industrialist, who joined the Western Electric Company, a manufacturer of telephone hardware for Bell Telephone. Bell's engineers had been trying to improve the reliability of the transmission systems. Because much of the amplification equipment was buried underground, there was an economic need to reduce the frequency of equipment failure and repair. Bell Telephone had already realized the economic importance of reducing variation in any manufacturing process, as well as the fact that continual adjustments to a process (in response to non-conformance) could actually increase variation and decrease quality. In a memorandum written on May 16, 1924, Shewhart was able to describe the problem in terms of two types of variation: "assignable-cause" and "chance-cause". ${ }^{8,9} \mathrm{He}$ introduced the "control chart" as a tool for distinguishing between the two. Shewhart stressed the importance of bringing a process into a state of "statistical control", where there was only chance-cause variation. ${ }^{8}$ Keeping the process under statistical control was

From the Department of Anesthesia, University of Calgary, Calgary, Alberta, Canada.

Address correspondence to: Dr. J.M. Davies, Department of Anesthesia, University of Calgary, Foothills Medical Centre, Calgary Health Region, 1403 - 29th Street NW, Calgary, Alberta T2N 2T9, Canada. Phone: 403-944-4707; Fax: 403-944-2425;

E-mail: jdavies@ucalgary.ca 
necessary for economic management and being able to predict future output. To Shewhart, the control chart was "the formulation of a scientific basis for securing economic control."

Shewhart further contributed to the scientific approach to quality, with his 1939 development of the four step cycle for improvement: 'Plan, Do, Check, Act - (PDCA) Repeat the Cycle' technique. Also known as the 'Shewhart Cycle' and the 'Deming Cycle', PDCA has since been renamed the 'Plan, Do, Study, Act' (PDSA) cycle. ${ }^{10}$

\section{How is SPC used?}

SPC requires collecting a numerator, or the number of occurrences of an event over a specified time, and a denominator. In anesthesia, the denominator would therefore be the number of anesthetics given during that time. The number of events will vary, in one of two ways. 'Natural' variation represents the normal noise inherent in any 'stable' process and can be defined by calculated probability limits, set to plus or minus three standard deviations. 'Unnatural' variation occurs outside the probability limits and results from an unstable process.

Once the variation of any process is determined, attempts are then made to improve the process. To do so requires answering the question: is the variation just noise or a signal of real change? ${ }^{11}$ Natural variation or noise is related to the underlying contributory factors to the process. For example, in Fasting and Gisvold's paper, moderate or serious difficulties with tracheal intubation was considered a stable process, with a detected rate of $1.5 \%{ }^{4}$ Any improvements to this aspect of care must focus on both the detection of problems (recognizing, reporting and recording), as well as the usual factors in such problems. These would include:

- The patient's anatomy and co-morbidities

- e.g., did the patient have a life-threatening condition?

- The training and experience of the personnel

- e.g., was extra help readily available?

- The equipment and environment in which the care was delivered

- e.g., was a difficult intubation tray or cart available, no matter where tracheal intubation was undertaken?

In contrast, unnatural variation is related to potentially new contributory factors and requires careful investigation. As described in Fasting and Gisvold's study, difficulties during emergence from general anesthesia represented an unstable process. Further examination revealed that, in fact, the data represented two stable processes separated in time by the results of a departmental intervention. ${ }^{4}$ Using this problem as an example, when reviewing unstable processes, consideration should be given to determining what other events have occurred during the same period. These events could include: educational activities, changes to the budget, mergers of hospitals and regions, changes in government policy, ${ }^{12}$ and even outbreak of an epidemic. ${ }^{13}$

\section{Why is SPC important?}

Fasting and Gisvold believe that because mortality and serious morbidity related to anesthesia are becoming "exceedingly rare", part of the quality of anesthetic care can - and should - be evaluated by reviewing "adverse events". ${ }^{4}$ These events are related to the process of anesthesia and have the potential to become part of the evolving development of an adverse outcome. ${ }^{14}$ Although some intraoperative adverse events occur frequently, the fewer their numbers, then the better the care. By using SPC, different anesthetic processes can be studied, their variation examined, and the occurrence of underlying adverse events reduced.

In fact, the potential for this and other statistical techniques in health care may have been anticipated by Florence Nightingale, who is credited with stating that "to understand God's thoughts we must study statistics, for these are the measure of His purpose".$^{15}$ Nightingale is also quoted as saying "the ultimate goal is to manage quality. But you cannot manage it until you have way to measure it, and you cannot measure it until you are able to monitor it." 16

Nightingale's era, that of the late 19th and early 20 th centuries, was characterized by development of a systematic approach to quality - to assist increasingly complex industrial growth. Similar developments occurred in healthcare. Ernest Hey Groves, an English surgeon, attempted to develop a systematic collection of records for the 73,000 major operations then performed annually. This collection was to be an "annual standard of reference and index of progress", allowing yearly comparison of results and improvement. Groves listed six points for his national registry:

- a central authority to collect and publish statistics;

- an organizing committee to detail nomenclature and registration;

- a uniform system of nomenclature of diseases and operations;

- recording of data under supervision of each hospital registrar;

- annual reporting of combined figures from 
each hospital; and

- late outcome recordings of cancer patients. ${ }^{17}$

Many years later, these six points could still be used to monitor practice. Similarly, application of SPC to healthcare is not new. One important and interesting application is that by Mohammed and colleagues, who used SPC to examine six cases studies, including pediatric cardiac surgery mortality in Bristol, mortality rates of convicted serial killer Dr. Harold Shipman, and mortality after fractured hips. ${ }^{18}$ In anesthesia, Eagle and Davies described use of the technique to the seemingly perennial problem of delay of the first operating room case of the day. ${ }^{16}$

Finally, review of adverse events is only one part of improving quality of anesthetic care. Fasting and Gisvold emphasize the importance of comparing results from their department with those published in the international literature, for example, for rates of difficulties with tracheal intubation. ${ }^{4}$ They also emphasize 'closing the quality circle' - ensuring that steps are taken to proceed from detection of a problem to its resolution. In doing so, they maintain the tradition of learning from others, at the individual and organizational level, ${ }^{19}$ and continuing to improve the safety of anesthetic practice.

\section{Apprendre des autres}

Au cours des 157 dernières années, la spécialité de l'anesthésie a évolué de façon impressionnante au plan sécurité. Ce progrès résulte de différents facteurs qui comprennent :

- l'invention de la seringue et de l'aiguille hypodermiques (1853)

- le développement de l'intubation endotrachéale (1880)

- la mesure et l'enregistrement de la tension artérielle peropératoire (1901)

- la conception du premier ordinateur électronique (1943). ${ }^{1}$

Chacun de ces facteurs est important et a contribué à améliorer les soins et l'évolution des patients. Important aussi en ce que chacun provient de l'extérieur de la spécialité de l'anesthésie. Par exemple, l'intubation endotrachéale (orale à l'aveugle) a été développée en 1880 par un chirurgien de Glasgow, William Macewen, ancien étudiant de Lister et pio- nnier en neurochirurgie. ${ }^{1-s}$ Le premier ordinateur électronique, ENIAC, a été mis au point pour calculer les tables de tir d'artillerie. ${ }^{1}$ Mais, malgré leurs origines et leurs inventeurs étrangers, ces perfectionnements ont été adoptés par les anesthésiologistes, dans certains cas comme les leurs.

Un autre exemple de l'adoption d'une technique venant d'une industrie différente est celle que décrivent Fasting et Gisvold dans le présent numéro du Journal. ${ }^{4}$ Ils présentent l'utilisation qu'ils ont faite de la technique du contrôle statistique du processus (CSP) pour quatre événements indésirables survenant pendant l'anesthésie : les difficultés d'intubation, l'anesthésie régionale (du plexus brachial) inadéquate, les erreurs de médicaments et les difficultés du retour à la conscience après une anesthésie générale. Même si les auteurs ont recueilli régulièrement les données sur toutes les anesthésies réalisées pendant 18 ans à Trondheim, en Norvège, ${ }^{5}$ ils n'ont appliqué le CSP qu'aux données recueillies prospectivement pendant les cinq dernières années.

\section{Qu'est que le CSP ?}

Le CSP est un terme construit à partir de trois mots employés de façon très précise. "Contrôle" fait référence au fait de garder quelque chose à l'intérieur de certaines limites ou de réguler l'activité de telle sorte que ses conséquences puissent être prédites avec un certain degré d'exactitude. "Statistique" fait référence aux nombres et à la formulation de conclusions à partir de nombres. "Processus" fait référence à une activité, par opposition au contrôle statistique de la qualité ou CSQ qui traite des caractéristiques d'un produit.

Ainsi, le CSP c'est l'usage de méthodes statistiques spécifiques pour analyser un processus et son évolution. Le CSP permet aux travailleurs de prendre les mesures appropriées pour réaliser et maintenir le processus à l'intérieur de certaines limites, c'est-à-dire, selon un contrôle statistique. Par conséquent, les travailleurs peuvent améliorer sans cesse le processus. ${ }^{7}$

Le CSP a été décrit pour la première fois par Walter A. Shewhart, un industriel américain, membre de la Western Electric Company, un fabricant de matériel téléphonique pour Bell Telephone. Les ingénieurs de Bell essayaient d'améliorer la fiabilité des systèmes de transmission. La plus grande partie du matériel d'amplification étant enterré, il y avait un impératif économique de réduire la fréquence de bris et de réparation. Bell Telephone connaissait déjà l'importance économique de réduire la variation de toute production, et le fait que les ajustements continus d'un procédé (en réponse à la non-conformité) puissent en 
réalité augmenter la variation et diminuer la qualité Dans un document de travail du 16 mai 1924, Shewhart a pu décrire le problème par deux types de variations : de "cause déterminante" et de "cause afférente". 8 "Il a présenté le "graphique de contrôle" comme un outil permettant de distinguer les deux. Shewhart a insisté sur l'importance de développer le processus jusqu'à un état de "contrôle statistique", où il n'y a qu'une variation de cause afférente. ${ }^{8}$ Il était nécessaire de garder le processus sous contrôle statistique pour la gestion économique et la capacité de prédire un futur rendement. Pour Shewhart, le graphique de contrôle était "la formulation d'une base scientifique pour s'assurer le contrôle économique."

Shewhart a contribué aussi à l'approche scientifique de la qualité par la mise au point, en 1939, de son cycle d'amélioration en quatre étapes : "Planifier, Faire, Vérifier, Agir (PFVA), Répéter le cycle". Connu également comme le "Cycle de Shewhart" et "Cycle de Deming", le PFVA a été renommé depuis le cycle PFEA ou "Planifier, Faire, Étudier, Agir".

\section{Comment utiliser le CSP ?}

Le CSP nécessite la collecte d'un numérateur, c'est-àdire du nombre d'occurrences d'un événement pendant un temps spécifique, et d'un dénominateur. En anesthésie, le dénominateur sera donc le nombre d'anesthésies pratiquées pendant ce temps. Le nombre d'événements peut varier de deux façons. La variation "naturelle" représente le bruit normal inhérent à tout processus "stable" et peut être définie par les limites de probabilité calculées, réglées à plus ou moins trois écarts types. La variation "anormale" survient en dehors des limites de la probabilité et provient d'un processus instable.

Une fois la variation du processus déterminée, on tente ensuite d'améliorer le processus. Pour ce faire, il faut répondre à la question : la variation est-elle seulement un bruit ou le signal d'un changement réel ? ${ }^{11} \mathrm{La}$ variation naturelle ou le bruit sont reliés aux facteurs contributifs à l'origine du processus. Par exemple, dans l'article de Fasting et Gisvold, des difficultés modérées ou sérieuses d'intubation endotrachéale étaient considérées comme un processus stable pour un taux décelé de $1,5 \% .{ }^{4}$ Toute amélioration de cet aspect de l'intervention doit porter d'abord sur la détection des problèmes (reconnaissance, notification et enregistrement), autant que sur les facteurs communs de ce genre de problèmes. Ils comprennent :

- L'anatomie du patient et les co-morbidités par ex., le patient présente-t-il une affection grave ?
- La formation et l'expérience du personnel par ex, du personnel supplémentaire est-il rapidement disponible ?

- Le matériel, l'environnement dans lequel les soins sont donnés

par ex., un plateau ou un chariot pour intubation difficile est-il disponible, peu importe où a lieu l'intubation ?

Par contre, la variation anormale est reliée à des facteurs contributifs potentiellement nouveaux et exige une investigation minutieuse. Ainsi que le décrivent Fasting et Gisvold, les difficultés du retour à la conscience après une anesthésie générale représentent un processus instable. Un examen plus poussé a révélé que, de fait, les données représentent deux processus stables séparés dans le temps par les résultats d'une intervention départementale. ${ }^{4}$ En utilisant ce problème comme un exemple, au moment de revoir les processus instables, on devrait chercher à déterminer quels autres événements se sont produits pendant la même période. Il pourrait s'agir, entre autres : d'activités pédagogiques, de modifications budgétaires, de regroupements d'hôpitaux et de régions, de changements de politique gouvernementale, ${ }^{12}$ et même de l'éclosion d'une épidémie. ${ }^{13}$

\section{Pourquoi le CSP est-il important ?}

Fasting et Gisvold croient que, étant donné que la mortalité et la morbidité grave reliées à l'anesthésie sont devenues "extrêmement rares", une partie de la qualité de l'anesthésie peut - et doit - être évaluée en passant en revue les "événements indésirables". ${ }^{4}$ Ces événements sont reliés au processus de l'anesthésie et ont le potentiel de faire partie du développement évolutif d'une complication. ${ }^{14}$ Même si certains événements peropératoires surviennent fréquemment, moins ils sont nombreux, meilleurs sont les soins. En utilisant le CSP, différents processus anesthésiques peuvent être étudiés, leur variation examinée et l'occurrence d'événements indésirables sous-jacents réduite.

De fait, le potentiel de cette technique statistique, et d'autres, en soins de santé a pu être envisagé par Florence Nightingale qui aurait déclaré que "pour comprendre les pensées de Dieu, nous devons étudier les statistiques, parce qu'elles sont la mesure de Son objectif". ${ }^{5}$ Nightingale a aussi dit que "le but final est de gérer la qualité. Mais vous ne pouvez la gérer sans avoir de moyen de la mesurer et vous ne pouvez la mesurer si vous ne pouvez la surveiller". ${ }^{16}$

L'époque de Nightingale, celle de la fin du $19^{\mathrm{e}}$ et du début du $20^{\mathrm{e}}$ siècles, a été caractérisée par le 
développement d'une approche systématique de la qualité visant à faciliter la croissance industrielle de plus en plus complexe. Des progrès similaires sont survenus dans les soins de santé. Ernest Hey Groves, un chirurgien anglais, a essayé de mettre au point une collecte systématique des dossiers de 73000 opérations majeures réalisées alors annuellement. Cette cueillette devait être une "norme annuelle de référence et un index de progrès" permettant une comparaison annuelle des résultats et de l'évolution. Groves énumère les six points de son registre national :

- une autorité centrale pour recueillir et publier les statistiques

- un comité d'organisation pour présenter la nomenclature et l'enregistrement

- un système uniforme de nomenclature des maladies et des opérations

- l'enregistrement de données sous supervision du préposé au registre de chaque hôpital

- le rapport annuel des chiffres combinés de chaque hôpital

- les dernières notes d'évolution des cancéreux. ${ }^{17}$

De nombreuses années plus tard, ces six points peuvent toujours servir à surveiller l'exercice de l'anesthésie. De même, l'application du CSP aux soins de santé n'est pas nouvelle. Une application importante et intéressante est celle de Mohammed et de ses collègues qui ont utilisé le CSP pour vérifier six études de cas, y compris une mortalité en chirurgie cardiaque pédiatrique à Bristol, les taux de mortalité du tueur en série reconnu, le Dr Harold Shipman et la mortalité après des fractures de hanche. ${ }^{18}$ En anesthésie, Eagle et Davies ont décrit l'application de la technique au problème apparemment permanent du retard à commencer la première opération du jour. ${ }^{16}$

Finalement, la revue des événements indésirables n'est qu'une partie de l'amélioration de la qualité des soins anesthésiques. Fasting et Gisvold insistent sur l'importance de comparer les résultats de leur service avec ceux qui sont publiés dans la documentation internationale, par exemple pour les taux de difficultés d'intubation endotrachéale. Ils insistent également sur le fait de "boucler la boucle" qui consiste à s'assurer que les étapes ont été suivies, de la détection à la résolution du problème. En agissant ainsi, ils maintiennent la tradition d'apprendre des autres, au plan individuel et organisationnel, ${ }^{19}$ et de continuer d'améliorer la sécurité de l'exercice de l'anesthésie.

\section{References}

1 Atkinson RS, Rushman GB, Alfred Lee J. A Synopsis of Anaesthesia, 8th ed. Bristol: John Wright and Sons; 1977.
2 MacEwen $W$. Clinical observations on the introduction of tracheal tubes by the mouth instead of performing tracheotomy or laryngotomy. Br Med J 1880; 2 :

122-4; 163-5. (Reprinted in Survey of Anesthesiology 1969; 13: 105-20).

3 Haridus RP. Awake blind oral intubation by internal palpation. Educational Synopses in Anesthesiology and Critical Care Medicine. The Online Journal of Anesthesiology 1996; 3: 2-7.

http://www.gasnet.org/esia/pdf/1996/esia9604.pdf (visited May 5, 2003).

4 Fasting S, Gisvold SE. Statistical process control methods allow the analysis and improvement of anesthesia care. Can J Anesth 2003; 50: 767-74.

5 Gisvold SE, Molang PO, Hallan S, Arnulf V. A study of risk evaluation and quality control in anaesthesia. Int J Risk Saf Med 1990; 1: 17-26.

6 Anonymous. QReview. Chapter 2. Basic Quality Concepts. Certified Quality Engineer. CQEWeb.com. http://www.cqeweb.com (visited May 2, 2003).

7 Gilmartin $R V$. America's revolution in health care: increasing competition and choice; improving people's health. February 27, 1997. Executive Speeches August/September 1997. The Executive Speaker Library, Speech 5721. http://www.executivespeaker.com/spch0021.html (visited 2 May, 2002).

8 Cutler AN. Biography of Walter A. Shewhart. Sigma Engineering Partnership.17 September, 2001. http://www.sigma-engineering.co.uk/light/shewhartbiog.htm (visited May 6, 2003).

9 Anonymous. Walter A. Shewhart. Father of statistical quality control. History. American Society for Quality. http://www.asq.org/join/about/history/shewhart.ht $\mathrm{ml}$ (visited May 6, 2003).

10 Berwick DM. Developing and testing changes in delivery of care. Ann Int Med 1998; 128: 651-6.

11 Anonymous. Michael L. McDonald \& Associates, Incorporated. Lesson 4. Control Charts. http:// www.mlmcdata.com/main.htm (visited May 6, 2003).

12 Dean $M$. UK government reveals plans to give hospitals more control. Lancet 2003; 361: 1110.

13 Anonymous. Infection control guidance for health care workers in health care facilities and other institutional settings. Severe acute respiratory syndrome (SARS).

(Revised May 5, 2003). http://www.hcsc.gc.ca/pphb-dgspsp/sars-sras/ic-ci/sars-icghcwotherinst_e.html (visited May 7, 2003).

14 Gaba DM, Maxwell M, DeAnda A. Anesthetic mishaps: breaking the chain of accident evolution. Anesthesiology 1987; 66: 670-6.

15 Anonymous. The Concise Oxford Dictionary of Quotations, 2nd ed. Toronto: Oxford University Press; 1986. 
16 Eagle CJ, Davies JM. Current models of "quality" - an introduction for anaesthetists. Can J Anaesth 1993; 40: 851-62.

17 Pollock A, Evans M. Surgical Audit. London:

Butterworths; 1989.

18 Mohammed MA, Cheng KK, Rouse A, Marshall T.

Bristol, Shipman, and clinical governance: Shewhart's forgotten lessons. Lancet 2001; 357: 463-7.

19 Senge PM. The Fifth Discipline. The Art and Practice of the Learning Organization. New York: Currency Doubleday; 1990. 